\title{
Effective Channels for Establishing an Irlen Centre to Help People with Reading Problems in Thailand
}

\author{
1Petcharat Lovichakorntikul, ${ }^{2}$ John Walsh* \\ 1,2School of Management, Shinawatra International University, Bangkok, Thailand \\ *jcwalsh@siu.ac.th
}

\begin{abstract}
Irlen Syndrome was identified in the 1980s as a condition that affects people who cannot see written text on a page properly. Consequently, their ability to learn in a standard educational setting is significantly limited and their future lives constrained. Further, affected people, in common with people with dyslexia, are often accused of lack of intellect or interest in studies and this can be a source of stress and relationship breakdown. The eponymous Irlen method provides a technical solution to the problem involving coloured lenses, which helps some people. Nevertheless, the approach falls between the educational and medical worlds and this provides some credibility issues. This paper examines the possibility of establishing an effective and profitable Irlen Centre in Thailand through personal, in-depth interviews with relevant professionals in a variety of fields. The findings are considered in the light of contemporary Thai culture and the pressure on young people to take an active part in a market-oriented society.
\end{abstract}

Keywords: Certified Irlen Diagnosticians, Certified Irlen Screeners, Irlen Syndrome, Irlen Centre

\section{Introduction and Literature}

Beginning in 1980, research directed by Irlen (1983) under a United States Federal Research Grant program studied methods of helping children and adults with reading and learning disabilities. During the period from 1981 to 1983, Ms. Irlen interviewed more than 1,500 adults with reading problems and classified these people into five groups of symptoms experienced (singularly or in combination), specifically: Light Sensitivity; Background Accommodation; Print Distortions; Restricted Span of Recognition; and Environmental Misperceptions. These are defined in the Irlen Reading Perceptual Scale (IRPS) Instructional Manual (Irlen, 2003). Individuals affected may find printed letters hazy, out-of-focus, differently sized or in other ways difficult to understand. In some cases, individuals are highly sensitive to bright or fluorescent lights or may have difficulty in coordinating their movements with moving objects such as escalators. This combination of symptoms is referred to as Irlen Syndrome. Formerly also known as Scotopic Sensitivity Syndrome, Irlen Syndrome is a visual perceptual dysfunction that has been found to be the root of reading difficulties for many individuals. It is brain based and triggered by sensitivity to a specific portion of the light spectrum. People who suffer from Irlen Syndrome are sensitive to certain wavelengths and frequencies of light which make them be unable to process information (Irlen, 2005).

Normally, Irlen Syndrome affects about one in six in the general population and almost half of all those diagnosed with dyslexia. Approximately 46\% of individuals diagnosed with reading difficulties, learning disabilities, attention problems or other behavioral problems may actually suffer from Irlen Syndrome. Individuals with Irlen Syndrome cannot read comfortably, they tend to experience eye fatigue and strain; painful or watery eyes; suffer fatigue, headaches, dizziness or nausea or else feel fidgety or restless. They may also be bothered by contrast, glare, fluorescent lights, bright lights, sunlight and sometimes lights at night. Reading, writing, attention and concentration are problematic (Irlen, 2005). In educational settings, they may be wrongly categorized as having below average IQ or aptitude for learning. Unflattering labels may be placed upon them by parents, teachers and colleagues and this can lead to long-term problems in obtaining and keeping employment, in family relationships and in quality of life. In general, these problems tend to run in families and are not currently identified by other standardized educational, visual, or medical tests. They can manifest themselves differently for each individual. It is not remediable and is often a lifetime obstacle for individuals with Irlen Syndrome to fight in learning and performances (Irlen, 2005). 
It was discovered that Irlen Syndrome is a perceptual or neurological disorder and not an optical problem. Several patients with Irlen Syndrome have Plano vision. It means that they have normal sight but they could not read as long as they would like, perhaps only a few seconds, before needing to take a break because of fatigue or eye strain or other health problem. They not only have all these physical symptoms, but also have poor reading rate, accuracy, and comprehension. However, this is not a vision problem (Ang, 2008). Irlen Centre trained professionals have been able to treat some patients through the use of coloured lenses and other means of mediating between the patient, the printed page and the external environment. This included altering the type and nature of light in which the patient tries to read. Some success has been achieved in the USA and elsewhere using this method and Centres have been opened in 45 different countries. A Centre was opened in Thailand in 2009 and a small number of patients were accepted for treatment. In common with private sector medical and quasi-medical facilities in Thailand, the Centre was planned to operate on a profitmaking basis and recover its costs at least in part from the patients or their family members.

Issues surrounding the payment for health care can be controversial and subject to contending political ideologies. Irrespective of personal beliefs and of political ideologies, it is evident that healthcare costs are increasing as improving lifestyles and anticipated life spans elevate aspirations for what medicine might achieve for individuals. However, these increased costs mean that healthcare is, increasingly, divided between those who can afford it and those who must do without. This has several implications. The first is that states that have tried to offer a more or less egalitarian access to all or at least many forms of healthcare are struggling with the imperative of reforming pricing and rationing decisions to make best use of available resources. This is true of China (e.g. Hu et al., 2008). In common with Communist and former Communist countries, Thailand is seeking to deal with the provision of decent quality health care to a range of people under the 30 baht health care scheme. This has proved problematic from the outset because of the dangers of a dual system, in which some practitioners and institutions opt out of public health provision for the sake of superior profits in the private sector, no matter how hard a government tries to ensure equitable access to services (Dorslaer et al., 2008). The problem is exacerbated in Thailand because of vertical differences between public and private sector provision. As part of the effort to make Thailand a hub for health tourism, a number of private sector hospitals in Thailand have upgraded their facilities and attendant costs significantly (Pachanee and Wibulpolprasert, 2006). The situation is further complicated by the nature of the Irlen treatment, because it has been contested in terms of validity and because it is not provided through mainstream health service facilities, even though ongoing research indicates that some positive effects have been demonstrated in experimental conditions (Cardona et al., 2010). Under such circumstances, there are concerns that pricing for some services will be predatory in nature (Ursic and Helgeson, 1994). These are additional fears than an Irlen Centre or its equivalent has to overcome in addition to advertising its existence to potential customers. The ways in which this can be tackled and the implications for Thai society of that attempt are the subject of this paper, in addition to the practical issue of the feasibility of establishing the centre in the first place.

\section{Research Questions}

The research follows two lines of investigation: commercial and cultural. From the commercial perspective, it was concerned to identify the ways in which a new product or service could be introduced into Thailand in a market sector that was little-known and with a high emotional component. Healthcare issues in general stimulate powerful emotional responses and there is a heightened need, therefore, for service providers to establish trust and understanding in the personal relationship with the patient and the patient's responsible family members. From the cultural perspective, the research sought to investigate the ways in which Thai culture and society has an impact upon an otherwise purely commercial decision such as opening and operating an Irlen Centre. Thai culture is generally considered to be group and family-oriented, with a stated desire for conformity, deference and obedience. It is also increasingly marketised as the impact of advanced capitalism encroaches upon society through the processes of globalization. These factors are rather stronger in action in the capital city Bangkok, which is larger and more significant in economic, political, cultural and electoral terms than the rest of the country, in which urbanization proceeds at a rather more modest pace. Are they relevant to the ways in which people might relate to a service such as the Irlen Centre? 


\section{Research Methodology}

Thailand's Irlen Centre was opened (in partnership with one of the researchers) in 2009 and remained open for only two months, owing to business partnership issues. While it was open, more than 200 questionnaires were distributed to potential patients for screening and assessment purposes. The name and nature of Irlen Syndrome were hitherto almost unknown in Thailand prior to this and there was little time to spread awareness and acceptance. Three clients did receive treatment, one of whom received two sessions and has subsequently gone on to a successful tertiary level educational career. However, while the Centre was open, it was possible to conduct a number of detailed, personal interviews with relevant professionals with a view to understanding the Thai system of medical service provision, the interaction between medical professionals and patients and the commercial aspects of running a medical facility in Bangkok. Interviews were accompanied by extensive note-taking and supplemented by ethnographic observation of potential patients and their guardians, in addition to analysis of secondary data relevant to the issues concerned.

\section{Data Analysis}

Primary research data was documented and entered into a database with the secondary data. The database as a whole was then subjected to a content analysis approach with a relational perspective. With such an approach, the attempt is made to identify the relationship between different concepts that were designated (through initial analysis and consideration) as being significant. A flexible approach was taken to identifying the types of relationship that might exist, since simple correlations, whether negative or positive, rarely explain human interactions accurately. Additional possibilities include non-linear, circular and teleological relationships.

Formal interviews were conducted with eight medical doctors, including specialists in cornea refractive surgery, one nurse, two instructors, three business owners/professionals, one government officer and parents and student clients of the Centre. A semi-structured interview agenda was prepared before the interviews were conducted, although each interview was constructed as a conversation in which respondents could investigate and elaborate upon areas of particular interest and relevance to them. Commonly explored areas of interest included:

1. Familiarity with Irlen Syndrome and its treatment

2. Relationship with the Syndrome and its impact upon individuals within a particular social context

3. Desired ways to access Irlen Centre services and channels of delivery: via teachers/instructors or medical specialists such as doctors, ophthalmologists and optometrists or some other approach

4. Payment issues

5. Additional societal and cultural issues relating to the Syndrome.

Extensive note-taking during the interviews, which were conducted in the Thai language, was accompanied by transcription as soon after the interview was completed as possible. Subsequent content analysis was conducted along the lines of conceptual analysis. The purpose of the interviewing was to answer the research questions specified above and to provide additional information and insights into the relationship between innovative health services entering the Thai market and the perceptions of stakeholders of those services.

\section{Findings}

\section{Perception of Irlen Syndrome in Thailand}

None of the respondents, even the medical professionals, had any awareness of Irlen Syndrome prior to the interviews. This was not altogether surprising and was known to have occurred in other markets prior to introduction of Centres. Having been acquainted with the symptoms of the condition, doctors could not recall any previous patient as having expressed similar symptoms or who might have been diagnosed positively. On the other hand, the medical professionals were very conversant with dyslexia and had been involved with treating such patients before. However, once having been acquainted with the Syndrome, respondents were 
generally supportive and willing to obtain and consider more information. It became, in short, very evident that there was a very low level of awareness of Irlen Syndrome in Thailand among not just the public but also the medical profession, which indicates the need to disseminate information at all levels of the chain of delivery - that is, at every stage at which patients could be identified as candidates for treatment and diagnosis at an Irlen Centre.

\section{Perspectives from Medical Doctors}

The medical doctors interviewed tended to be specialists in their own fields and to be unaware of developments in other fields, even if they were related fields. If the Irlen Syndrome symptoms were not directly relevant to their own specialty, therefore, they tended to show little interest in learning more about them, preferring instead to rely on the referral system, which would pass a patient on to another professional. An opinion was expressed that more co-operation should be fostered between different branches of the education and public health system, including the schools, inspectors and peripatetic health officials. Doing so, of course, would require considerable efforts to promote information by the Irlen practitioners, who would also wish to work through and with the medical science programs of leading universities and in the popular media. There is an issue involved here concerning the relationship between professionals and potential patients and the receipt of information about a process which is neither properly educational nor medical in nature. There would be a further bureaucratic issue at the governmental level concerning which ministry and department would take responsibility for Irlen activities and related revenue concerns.

\section{Perspectives from Educators}

Educators were receptive to more information about Irlen Syndrome and its treatment. Experienced educators had anecdotes of previous students who seemed to be highly intelligent and motivated but whose examination and written assignment performance was continually below expectations. Those students were usually unable to reach their potential but the educators had little idea of what more they could do for them. Understanding the Irlen symptoms could provide another possible means of helping some students who might not otherwise be able to succeed. They agreed generally that educators had a close enough knowledge of most of their students (whom they knew for long enough) to be able to identify when Irlen-like problems might be manifested. Further, being aware of the issue might enable them to be more pro-active in spotting problems before they intensified as written exams proved more important in a student's career.

\section{Personal Experiences}

In terms of personal experiences, those respondents who had received treatment and their relatives were pleased with the treatment and appreciative of the fact that it appeared to offer a solution to a problem which had seemed difficult even to define. To some extent, the improvement in personal relationships this enabled was as valuable as the physical assistance provided by treatment. From a commercial marketing point of view, of course, this element offers perhaps the vital means of appeal to potential clients: the problem that seems to spoil domestic tranquility and has no solution but can be resolved by technical means is a dream for many people.

However, there appears to remain some suspicion among certain people concerning the ability of Irlen practitioners to make the difference offered and of the nature of the problem in the first place. Once individuals have determined that there is a problem with a family member and then invested emotional energy into that decision, it can be difficult for them to accept that a comparatively simple technical adjustment can prove their whole opinion to be wrong. This again has implications for the marketing of Irlen Centres since it requires special effort to be devoted to reassurance, in addition to information provision, as a means of making sure that all relevant stakeholders are willing to buy into the concept. This, of course, is not helped by the fact that, until the present, medical professionals do not have any information to provide the stakeholders and cannot assuage any concerns that they might have. 


\section{Revenue Issues}

When asked, respondents replied that the total cost of Irlen treatment should be around 10-30,000 Baht (approximately US\$300-1,000). Although the costs suggested did vary, it is evident that the ideas presented have little relation with the actual cost of provision. The cost suggested is roughly equivalent to a course of dental treatment (e.g. a brace or retainer) or else a minor surgical operation at a Bangkok private sector hospital. Medical professionals suggested that the cost per visit should be around 500-1,000 Baht but, if a medical eyesight condition had been diagnosed, then as much as 3-5,000 Baht per visit might be appropriate. Again, the pricing suggested had little to do with the actual cost of provision but, instead, with what expectations of middle class people might be and what they would, as a consequence, be willing and able to pay. It was an unspoken assumption of the research that this kind of treatment would not be provided for the poor or working classes. It seems unlikely that Irlen Syndrome treatment will be adopted by the government for inclusion in the 30 Baht health care scheme in the foreseeable future.

\section{Creating Public Awareness}

It was generally considered that Thai people were stoical about facing illness and would stay away from doctors and hospitals unless really necessary. To some extent it can be considered that this resulted from the costs attached to medical care, since the welfare state has only been developed to a rudimentary extent in the Kingdom. Since the symptoms of Irlen Syndrome tend to be minor in them and are likely to go away if the attempt to read is ceased, then people are more likely to ignore the problem in the hope that it is temporary and will somehow disappear at some stage. It is all the more important, therefore, insofar as this anecdotal evidence is true, that more information is distributed throughout society so that those individuals who might be affected will be able to understand their vulnerability and the fact that they are not just facing transitory phenomena. Placing the information in a suitable format in schools, therefore, is of considerable importance. This raises an ethical issue faced by all societies in which medical services are available only from the private sector in that commercial enterprises providing information to children, their parents and teachers with a view to encouraging them to spend money is at best problematic. Providing information at some of the numerous education fairs and open days throughout the country might be a more useful way of operating.

However, the medical industry in Thailand has become very competitive at the high end and this is reflected in the advertising and marketing of goods and services. It is not, on the whole, the optimal time to launch a new healthcare product, even if actual interest in good health and the desire for high levels of educational attainment are as high as have ever been.

\section{Effective Channels}

Different respondents suggested alternative channels for delivery of Irlen Syndrome services. These were through educational institutions and health institutions. In terms of education, the international schools in Bangkok principally would be suitable conduits for providing information and services to young people. Such schools are not only full of pupils from affluent families with a desire to have their offspring reach the highest possible level but, also, teachers and ancillary staff willing to keep abreast of all developments in the sphere of education so as not just to assist pupils to achieve but also to maintain average grades and performance by dealing with under-performing pupils in one way or another. At international schools, of course, people from many different countries are represented and in at least some of these countries knowledge and understanding of Irlen Syndrome will be more developed and this will be helpful in disseminating knowledge to the relevant stakeholders.

In terms of health institutions, respondents suggested several different possible routes for provision of services, including hospitals and clinics. It is possible for independent, standalone clinics to offer services based on referrals but this brings about the problem of information to those who would be responsible for providing such referrals. In some cases, it would be possible for Irlen providers to rent or lease space within a hospital and distribute information from there, while also establishing relationships with medical professionals likely to come into contact with potential patients and clients. It remains the case, according to 
respondents, that Thai people will take advice from doctors when they do determine they need assistance and so the channel of delivery should include such doctor whenever possible.

\section{The Feasibility of Establishing an Irlen Centre in Thailand}

Respondents felt that an Irlen Centre might be feasible for Thailand but, because of the costs likely to be involved, only in Bangkok and almost certainly in association with an international school or private sector hospital. Some schools have special units for children with special learning needs such as because of dyslexia or Down's syndrome and this model might be appropriate for replication. The international school community in and around Bangkok is in fact quite small since strong networks link teachers, administrators, parents and pupils and information is, therefore, swiftly circulated. Such networks can, of course, be used as marketing tools.

However, the establishment of such a Centre would inevitably be a high-risk project because there is such a shortage of necessary information concerning the number and nature of possible clients, the ways of gaining access to them and the attractiveness of the service. Applying lessons learned from the establishment of Centres in other countries is only of limited value because cultural issues apply when it comes to medical concerns and the ways in which these are diagnosed. In any case, it is apparent that a long-term investment will be required because it will take a period of time probably measured in years rather than months for the appropriate information to be disseminated thoroughly throughout the identified channels.

\section{Irlen Syndrome and Thai Culture and Society}

Although Thailand has, economically, entered the capitalist stage of societal development, it remains languishing in the feudal period of political development in a number of ways. That has had the effect of preventing market-based transactions from dominating all forms of economic exchange and, instead, leaving social relations as being of primary importance. This is especially true of the relations between doctors and patients: Thai students pass through a national level of university examinations (in addition to some direct entrance programs) and the very highest ranked students are those permitted to enter medical school. As might be expected, not all medical doctors have a lifelong vocation, therefore, and a number have entered into different elements of Thai society, including politics. It has become common, therefore, for medical doctors to be regarded as gurus in whatever field of endeavour they might find themselves. It is clearly important for positive relationships to be established with the doctors if providers of quasi-medical goods and services are to be successful in setting up commercial enterprises.

The relationship is likely to be complicated somewhat by the nature of the Irlen Syndrome diagnosis and its methods of treatment. Any medical or educational issue that may be related to mental health is susceptible to association with undesirable taboos. Such an association can intensify the emotional component of what might otherwise be a purely professional or commercial relationship. Marketers need to be very sensitive, therefore, to the relationships that exist with all important stakeholders, especially given the nature of the client, who is very likely to be a young person referred by another professional or else though rumor or market-based marketing information. Other stakeholders will have perhaps intense emotional involvement in the treatment in one way or another and this is something the Irlen representative will need to be able to manage. As Thai society tends to be more group-oriented than individual-oriented, it might be possible to utilize the positive aspects of group dynamics in this respect. A positive feeling engendered by having a trustworthy and respected member of society involved in promoting the service would be likely to be a positive aspect.

\section{Conclusion}

This paper has addressed the connection between the practical and emotional-cultural issues involved with establishing a quasi-medical service centre in Thailand concerning a technique that is little known in any market. Through a program of in-depth personal interviewing, it was found that while respondents believed that any form of treatment that improves the quality of life of the patient-clients concerned would be warmly 
welcomed, it was also evident that this would be a high risk strategy because of the need to disseminate information for a lengthy period of time before referrals could be expected on any meaningful scale. Marketing the putative Centre would require a measure of word-of-mouth and personal recommendations and these means are always difficult for people to control.

The contribution of this research to knowledge is centered on the relationship between the new entry of medical services into the Thai market and the opinions and perceptions of important stakeholders of those services. This has taken place in the context of comparatively highly priced medical services which are far above the aspiration of the great majority of Thai people to afford. Few studies of Thai society have sought to relate the feasibility of opening a new facility to the reception it is likely to receive from possible customers and other stakeholders. Most considerations of the expansion of capitalism in the Kingdom has focused on the impact of the commercialization and marketization of local institutions and facilities rather than the entrance of new opportunities, however problematic they might appear to some. The openness of the stakeholders interviewed was an issue of interest. On the other hand, the study is limited by the slender nature of the sample available that had actually passed through the treatment. This is in addition to the usual issues of time and space that are endemic to a study of this sort. Longitudinal study or research in another country in which Irlen services are available (e.g. Singapore) would help to broaden the findings of the current research.

The ability to make recommendations in the present case is limited by the decision that has already been taken to discontinue to the service in Thailand, at least under the present management. It can be said that the market appears open to new entrants and that potential distribution channels do exist that when innovations do become available. Of course, there are incentives in the form of possible profits to be made in a private health system. There is scope for the government to take a lead in registering and guaranteeing new services as being appropriate for consumption. Success has been achieved in terms of regulating elective cosmetic surgery practices in the country and this indicates the competency is present.

\section{References}

Ang, D. (2008). Achieve Your Breakthrough in Learning Difficulties. Irlen Organization: Long Beach, California. Available at: http://www.irlen.com.sg/IRLEN.pdf.

Cardona, G., Borràs, R., Peris, E., and Castañé, M. (2010). A Placebo-Controlled Trial of Tinted Lenses in Adolescents with Good and Poor Academic Performance: Reading Accuracy and Speed. Journal of Optometry. 3(2): 94-101.

Doorslaer, E., Clarke, P., Savage, E. and Hall, J. (2008). Horizontal Inequities in Australia's Mixed Public/Private Health Care System. Health Policy, 86(1): 97-108.

Hu, S., Tang, S., Liu, Y., Zhao, Y., Escobar, M. and Ferranti, D. (2008). Reform of How Health Care is Paid for in China: Challenges and Opportunities. The Lancet, 372: 1846-53.

Irlen, H. (1983): Successful Treatment of Learning Disabilities, Paper presented at the 91 Annual Convention of the American Psychological Association, Anaheim, California.

Irlen, H. (2003). Irlen Reading Perceptual Scale. Irlen Organisation: Long Beach, California.

Irlen, H. (2005). Reading by the Colors: Overcoming Dyslexia and Other Reading Disabilities through the Irlen Method, revised edition. Perigee Trade: New York.

Pachanee, C. and Pibulpolprasert, S. (2006). Incoherent Policies on Universal Coverage of Health Insurance and Promotion of International Trade in Health Services in Thailand. Health Policy Planning, 21(4): 310-8.

Ursic, M. L. and Helgeson, G. J. (1994). Using Price as a Weapon: An Economic and Legal Analysis of Predatory Pricing. Industrial Marketing Management, 23(2): 125-31. 